\section{Long-term survival of two dogs after mitral valve plasty}

\author{
Isamu Kanemoto, ${ }^{1}$ Daisuke Taguchi, 1,2 \\ Kippei Mihara, ${ }^{1}$ Koudai Kawase, ${ }^{1,3}$ \\ Keiichi Sato, ${ }^{1}$ Kazuya Iwata, ${ }^{4}$ \\ Kouji Iwanaga 5
}

1Chayagasaka Animal Hospital, Nagoya, ${ }^{2}$ Green Animal Hospital, Sannohe;

3Sapporo Night Animal Hospital

Emergency and Critical Care, Sapporo;

${ }^{4}$ Iwata Animal Hospital, Gihu;

5Tokyo Veterinary Cardiology Center,

Meguro, Japan

\begin{abstract}
Two small 9-year-old dogs received mitral valve plasty (MVP) for severe mitral regurgitation that could not be controlled via drug administration. MVP consisted of chordal reconstruction using expanded polytetrafluoroethylene (ePTFE) sutures and semicircular suture annuloplasty using polypropylene sutures. In both cases, the clinical signs dramatically improved after MVP, although postoperative echocardiography revealed slight residual mitral regurgitant flow. Both dogs survived for several years after MVP without any signs of cardiac distress; one dog survived for 9 years and 1 month, and the other dog survived for 7 years and 10 months. These findings highlight the durability of ePTFE sutures as artificial chordae and the excellence of semicircular suture annuloplasty in small dogs.
\end{abstract}

\section{Introduction}

Mitral regurgitation (MR) caused by degenerative valve disease is the most frequent heart disease in old small-breed dogs. ${ }^{1}$ There are two surgical treatments for MR: mitral valve plasty (MVP), which repairs the valve, and mitral valve replacement (MVR), which replaces the valve with an artificial one. 2,3 At present, MVP is the preferred treatment because it improves quality of life; however, a repaired valve is less durable than a replaced valve. MVP techniques ${ }^{4}$ in humans include chordal reconstruction (CR), ${ }^{5}$ annuloplasty (AP),, 6 and valvuloplasty (VP); ${ }^{7}$ for CR and $\mathrm{VP}, 8,9$ but not suture AP, 10 the long-term results and materials used have been essentially established. Although there are some clinical reports of MVP in dogs, ${ }^{11-17}$ only one describes long-term survival after MVP. 18

We previously reported five cases of
MVP in small-breed dogs. ${ }^{14}$ In one case, the dog survived for 9 years and 1 month after MVP, and in another, the dog survived for 7 years and 10 months after MVP. Neither dog experienced cardiac distress after MVP, and both dogs were followed up until they died. In this report, we describe the preoperative examinations, MVP surgical procedures, and postoperative courses in these two dogs.

\section{Case Report \#1}

A male, 3.0-kg, 9-year-old Maltese received surgery for severe MR [American College of Veterinary Internal Medicine (ACVIM) stage D] at our hospital. He was initially diagnosed with moderate $\mathrm{MR}$ (ACVIM stage C) and had received an angiotensin-converting enzyme inhibitor (ACEI) since he was 8 years of age. Despite receiving multiple high-dose drug therapies, he had a sudden dyspnea attack 3 months before surgery and was repeatedly hospitalized owing to refractory pulmonary edema. Thoracic radiography performed during his first visit to our hospital revealed an enlarged heart [vertebrate heart score (VHS), 12.3v; cardiothoracic ratio (CTR), $62 \%$ ] with pulmonary edema. An echocardiogram revealed severe mitral regurgitant flow due to elongated and ruptured chordae tendineae, a high left ventricular internal dimension-diastole (LVIDd) of $32.7 \mathrm{~mm}$, a left atrium-to-aorta (LA:Ao) ratio of 2.5, and mild tricuspid regurgitant flow (Table 1). MVP was performed in accordance with the owner's request.

\section{Case Report \#2}

A male, 6.5-kg, 9-year-old Shi Zhu received surgery for severe MR (ACVIM stage D) at our hospital. He had had a grade $3 / 6$ systolic murmur since he was 7 years of age and had been receiving an ACEI and spironolactone. He experienced syncope at the age of 8 years, and carvedilol, furosemide, digoxin, isosorbide, and pimobendan were added to his drug regimen owing to severe coughing. Thoracic radiography performed during his first visit to our hospital revealed an enlarged heart (VHS, 11v; CTR, 74\%) and pulmonary congestion. An echocardiogram revealed severe mitral regurgitant flow due to elongated and ruptured chordae tendineae, a high LVIDd (40.0 mm), an LA:Ao ratio of 2.6, and mild tricuspid regurgitant flow (Table 1). MVP was performed in accordance with the owner's request.
Correspondence: Isamu Kanemoto, Chayagasaka Animal Hospital, 1-1-5 Shinnishi, Chikusa, Nagoya, Aichi, 464-0003 Japan.

Tel.: +81.52.773.1866 - Fax: +81.52.773.7488 E-mail: kanemoto@ta2.so-net.ne.jp

Key words: mitral valve plasty, chordal reconstruction, semicircular suture annuloplasty, long-term survival, small dog.

Acknowledgements: we would like to thank Editage (www.editage.jp) for English language editing.

Contribution: the authors contributed equally.

Received for publication: 3 December 2015. Accepted for publication: 18 December 2015.

This work is licensed under a Creative Commons Attribution NonCommercial 4.0 License (CC BY-NC 4.0).

(C) Copyright I. Kanemoto et al., 2017

Licensee PAGEPress srl, Italy

Veterinary Science Development 2017; 7:6335 doi:10.4081/vsd.2017.6335

\section{Anesthesia and open heart methods}

Deep surface-induced hypothermia under isoflurane anesthesia and low-flow rate cardiopulmonary bypass $(\mathrm{CPB})$ with a small-volume prime circuit were performed as reported previously. ${ }^{15}$ After anesthesia was induced, a venous cannula was inserted from the left jugular vein into the right atrium, and an arterial cannula was inserted through the left carotid artery toward the heart in right lateral recumbency. After CPB was prepared, thoracotomy was performed via the left fifth intercostal space. After CPB was started, the mitral valve was approached via an incision in the left atrium that was dorsal to the coronary groove. Valve leaflets and the chordal apparatus were inspected by using a nerve hook. After MVP, valve competence was evaluated via cold saline infusion into the left ventricle. The left atrium was closed by suturing in a simple continuous pattern. CPB discontinuation and postoperative care were performed as described previously. 15

\section{Surgical methods}

The basic MVP procedure consisted of $\mathrm{CR}$ in the anterior valve leaflet and semicircular suture AP in the mitral annulus (Figure 1). For CR, expanded polytetrafluoroethylene (ePTFE) sutures (CV-6, GoreTex) with needles at both ends were used. The sutures were sewn and fixed in the shape of a cross at the base of the ruptured 
chorda in the papillary muscle. Sutures were also applied in a scroll-like pattern from the ventricular side to the atrial side on both sides of the ruptured (or elongated) chorda in the anterior valve leaflet. Both sets of sutures were ligated after determining the length of the artificial chordae. As a result, one ruptured (or elongated) chorda was replaced by two artificial chordae (Figure 1A). For semicircular suture AP (the modified De Vega method), polypropylene (Proline) sutures (5-0, Ethicon) with needles at both ends and a pledget in between were used. The sutures were alternately sewn in a semicircular pattern in the mitral annulus of the posterior leaflet from the anterior to the posterior commissure site on both bases of the anterior leaflet; they were attached with a pledget and ligated by inserting a sizer with the same diameter as the anterior leaflet area into the valvular orifice (Figure 1B).

In case 1 , three ruptured chordae at A1, A1-2, and A2-3, respectively, in the anterior leaflet were reconstructed, and the diameter of the mitral annulus was reduced from 21 to $13 \mathrm{~mm}$ using semicircular suture AP. Mitral regurgitation almost disappeared, as assessed via a leak test. In case 2 , two ruptured chordae at A1-2 and A2-3, respective$1 y$, in the anterior leaflet were reconstructed, and the diameter of the mitral annulus was reduced from 26 to $15 \mathrm{~mm}$ using semicircular suture AP. On the basis of the results of a leak test, VP with two Proline 6-0 sutures was performed to directly suture the valve scallops at P1 and P2, and Proline 5-0 sutures attached to two pledgets were added via anterior commissure suture AP (the Kay method) (Figure 2).

\section{Postoperative course}

In both cases, auscultation revealed a Levine 2/6 systolic murmur, and echocardiography revealed slight mitral regurgitant flow after the operation. However, the clinical signs were dramatically improved (ACVIM B2 in both cases). After their discharge from our hospital, the dogs' personal doctors prescribed only ACEI.

As shown via thoracic radiography, the VHS in case 1 decreased from $12.3 \mathrm{v}$ (preoperative) to $10.9 \mathrm{v}$ (3 months postoperative) and was $11 \mathrm{v} 8$ years and 7 months after the operation (Table 1, VHS). On an echocardiogram, the mildly high luminance of the artificial chordae indicated good flexibility. Slight mitral regurgitant flow remained 3 months after the operation, and mild MR was noted 8 years and 7 months after the operation (Table 1, MR). The LVIDd decreased from $32.7 \mathrm{~mm}$ (preoperative) to $26.8 \mathrm{~mm}$ (3 months postoperative) and was $27.4 \mathrm{~mm} 8$ years 7 months after the operation (Table 1, LVIDd). Tricuspid regurgitant flow increased moderately. The dog in case 1 died at the age of 18 years and 1 month owing to senility; he survived for 9 years and 1 month after MVP.

As shown via thoracic radiography, the

VHS in case 2 decreased from $11 \mathrm{v}$ (preoperative) to $9.6 \mathrm{v}$ ( 3 months postoperative) and was $9.0 \mathrm{v} 5$ years and 2 months after the operation (Table 1, VHS). On an echocardiogram, the mildly high luminance of the artificial chordae indicated good flexibility.

Table 1. Preoperative and postoperative echocardiography and thoracic radiography findings.

\begin{tabular}{|c|c|c|c|c|}
\hline & Pre-op & Post-op 3m & Post-op 5y 2m & Post-op 8y 7m \\
\hline $\begin{array}{l}\text { MR } \\
\text { case } 1 \\
\text { case } 2\end{array}$ & $\begin{array}{l}\text { severe } \\
\text { severe }\end{array}$ & $\begin{array}{l}\text { slight } \\
\text { slight }\end{array}$ & $\begin{array}{c}- \\
\text { disappeared }\end{array}$ & $\begin{array}{c}\text { mild } \\
-\end{array}$ \\
\hline $\begin{array}{l}\text { TR } \\
\text { case } 1 \\
\text { case } 2\end{array}$ & $\begin{array}{l}\text { mild } \\
\text { mild }\end{array}$ & $\begin{array}{l}\text { mild } \\
\text { mild }\end{array}$ & moderate & moderate \\
\hline $\begin{array}{l}\text { LVIDd } \\
\text { case } 1 \\
\text { case } 2\end{array}$ & $\begin{array}{c}32.7 \\
40\end{array}$ & $\begin{array}{c}26.8 \\
28\end{array}$ & - & $\begin{array}{c}27.4 \\
-\end{array}$ \\
\hline $\begin{array}{l}\text { VHS } \\
\text { case } 1 \\
\text { case } 2\end{array}$ & $\begin{array}{c}12.3 \\
11\end{array}$ & $\begin{array}{c}10.9 \\
9.6\end{array}$ & - & $\begin{array}{l}11 \\
-\end{array}$ \\
\hline
\end{tabular}

Mitral regurgitation (MR) and tricuspid regurgitation (TR) were determined as semiquantitative volumes in Doppler images. LVIDd: left ventricular internal dimension-diastole (mm), VHS: vertebral heart scale (v), y: year, m: month, pre-op: preoperative, postop: postoperative. indicates that no work was done.
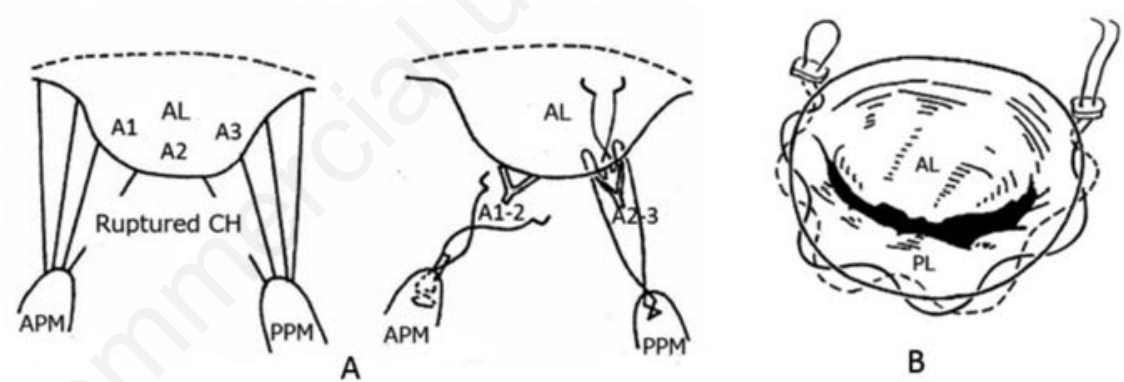

Figure 1. Basic mitral valve plasty techniques (chordal reconstruction and semicircular suture annuloplasty). A) Chordal reconstruction. A preoperative diagram is shown on the left, and a perioperative diagram is shown on the right. B) Semicircular suture annuloplasty (modified De Vega method). AL: anterior leaflet (A1, A2, A3), PL: posterior leaflet, $\mathrm{CH}$ : chordae, APM: anterior papillary muscle, PPM: posterior papillary muscle.
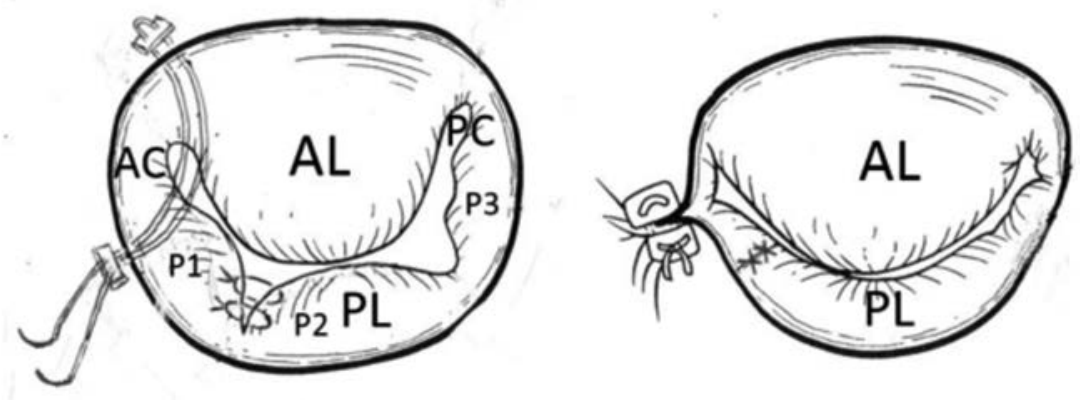

Figure 2. Additional mitral valve plasty techniques (posterior scallop direct suture and the Kay method). The prolapsed P1 and P2 scallops in the posterior leaflet (PL) were sutured directly using two 6-0 Proline sutures. Mattress sutures with a pair of pledgets were placed at the anterior commissure for localized residual regurgitation (Kay method). AL: anterior leaflet, AC: anterior commissure, PC: posterior commissure. P1, P2, and P3 refer to the posterior scallops in the PL. 
Slight mitral regurgitant flow remained 3 months after the operation and had disappeared completely by 5 years and 2 months after the operation (Table 1, MR). The LVIDd decreased from $40 \mathrm{~mm}$ (preoperative) to $28 \mathrm{~mm}$ ( 3 months postoperative) and was $21 \mathrm{~mm} 5$ years and 2 months after the operation (Table 1, LVIDd). Tricuspid regurgitant flow increased moderately (Table 1, TR). The dog in case 2 died at the age of 16 years and 11 months owing to senility; he survived for 7 years and 10 months after MVP.

\section{Discussion and Conclusions}

There are four types of MR lesions in humans as described by Carpentier et al. ${ }^{4}$ In dogs, most MR lesions are type II, which result from myxomatous degeneration accompanied by type I lesions caused by secondary enlargement of the annulus. ${ }^{1}$ Therefore, treatment of MR requires a combination of MVP techniques tailored to each lesion type, especially in small dogs, as done in humans.

There are three main MVP methods: 1) CR for ruptured or elongated chordae tendineae, 2) AP for a dilated mitral annulus to increase the coaptation area of the anterior and posterior leaflets, and 3) VP for deformed or redundant valve leaflets. Kanemoto et al. ${ }^{11}$ reported the first successful performance of MVP using VP (the McGoon method) ${ }^{7}$ combined with posterior commissural AP (the Kay method) ${ }^{19}$ in a small dog. In that case, MR was moderate and due to elongated chordae in the anterior leaflet. Both dogs in our report had severe MR due to ruptured chordae, and two MVP methods were used: CR with ePTFE sutures, and semicircular suture AP with Proline sutures.

Early CR for ruptured or elongated chorda tendineae involved the use of pericardium, silk, nylon, Teflon, or Dacron sutures, which have since been abandoned because of calcification and deterioration. ${ }^{20}$ CR using ePTFE sutures was subsequently developed in $1980^{5}$ and is now a standard MVP treatment for humans with MR. ${ }^{9} \mathrm{We}$ studied the use of ePTFE sutures as artificial chordae in dogs and presented our findings at the 20th World Small Veterinary Association Congress in 1995.21 Since then, we have used these sutures as artificial chordae in small dogs, 14,15 and in this study, we demonstrated their long-term durability as artificial chordae in two dogs, as have other studies.13,16-18 The most important aspect of the CR technique is the length of the artificial chordae. We determined that this length should be the same as the position of the mitral annulus.

There are two main techniques for AP for a secondary dilated mitral annulus, namely, suture AP and ring AP. Suture AP was developed early on and is primarily classified as commissural AP19 or semicircular suture AP. ${ }^{22,23}$ The recently developed ring AP uses an artificial ring 4,6 and is considered the gold standard MVP technique in humans. Rigid or flexible prosthetic rings allow optimal annular stabilization, but have some functional disadvantages including inhibition of physiological annular dynamics, systolic anterior motion of the anterior leaflet causing left ventricular outflow tract obstruction, and the potentiality of thrombus or pannus formation or hemolysis. Most of these drawbacks theoretically do not apply to suture AP, although recurrent mitral annular dilatation due to technical failures was initially reported for semicircular suture AP. 10

Semi-circular suture AP14,15,18 and partial ring AP using ePTFE patch material ${ }^{13,16}$ have been used to treat MVP in dogs. However, only one report showed longterm survival after performance of AP. ${ }^{18}$ In that report, which described three small dogs, semicircular suture AP and CR with ePTFE sutures were used, as done in the study herein. One dog died 2 years after surgery owing to dehiscence of the sutures in the mitral annulus tissue, which must be avoided in all AP methods. The other two dogs survived for more than 5 years, although mitral stenosis persisted in one.

Ring AP may be inappropriate for MVP in small dogs because the ring disturbs blood inflow by acting as a relatively largesized dike and causes hemolysis when regurgitant flow remains after MVP. ${ }^{24}$ Uechi et al. 16 found that soft prosthetic band AP with ePTFE sheets (Gore-Tex), used as a substitute for the pericardium in small dogs, caused thrombosis after surgery, as may ring AP. Therefore, we have used semicircular suture AP with Proline 5-0 sutures to repair a secondary dilated mitral annulus in small and toy dogs. In these cases, anticoagulant therapy was not always performed after the operation. We considered the size of the dilated mitral annulus to be adequately reduced when it was the same as that of the anterior leaflet area; use of this metric did not cause mitral stenosis. ${ }^{18}$ In both of our cases, the diameter of the mitral valve orifice was the same during and after the operation as assessed via echocardiography, which indicates that mitral annulus redilatation and stenosis did not recur. Therefore, semicircular suture AP appears to be an effective means of repairing a dilated mitral annulus in small dogs.
We used our basic MVP technique (CR and semicircular suture AP) in case 1, which involved lesions in only the anterior leaflet, and the dog survived for 9 years and 1 month after the operation. However, in case 2, in which severe MR was complicated by the presence of multiple lesions in both leaflets, two additional techniques were required: valve scallop suture VP (performed in the posterior leaflet to directly suture two neighboring prolapsed posterior valve scallops) and anterior commissure AP. As a result, mitral regurgitant flow was minimal at 3 months and absent at 5 years and 2 months after the operation. The dog in case 2 survived for 7 years and 10 months after the operation. We previously performed MVR in a small dog with severe, complicated MR (as in the dog in case 2) that died from thrombosis 2 years and 1 month after the operation. ${ }^{25}$ It appears that MVP consisting of CR with ePTFE sutures and semicircular suture AP with Proline sutures improves the long-term durability and usability of repaired valves compared with replaced valves in small dogs.

\section{References}

1. Kittleson MD, Kienle RD, eds. Myxomatous atrioventricular valvular degeneration. In: Small animal cardiovascular medicine. Kimberton: Mosby; 1998. pp. 297-318.

2. Gallegos RP, Gudbjartsson T, Aranki S. Mitral valve replacement. In: Cohn LH, ed. Cardiac surgery in the adult. 4th ed. New York: McGraw-Hill Medical; 2012. pp. 849-876.

3. Kouchoukos NT, Blackstone EH, Hanley FL, Kirklin JK, eds. Mitral valve disease. In: Kirklin/Barratt-Boyes Cardiac Surgery. 4th ed. Philadelphia: Elsevier Saunders; 2013. pp. 484-536.

4. Carpentier A, Deloche A, Dauptain J, et al. A new reconstructive operation for correction of mitral and tricuspid insufficiency. J Thorac Cardiovasc Surg 1971;61:1-13.

5. Frater RW, Vetter HO, Zussa C, et al. Chordal replacement in mitral valve repair. Circulation 1990;82:125-30.

6. Duran CG, Ubago JL. Clinical and hemodynamic performance of a totally flexible prosthetic ring for atrioventricular valve reconstruction. Ann Thorac Surg 1976;22:458-63.

7. McGoon DC. Repair of mitral insufficiency due to ruptured chordae tendineae. J Thorac Cardiovasc Surg 1960;39:357-62.

8. Carpentier A, Chauvaud S, Fabiani JN, 
et al. Reconstructive surgery of mitral valve incompetence. Ten-year appraisal. J Thorac Cardiovasc Surg 1980;79: 338-48.

9. David TE, Omran A, Armstrong S, et al. Long-term results of mitral valve repair for myxomatous disease with and without chordal replacement with expanded polytetrafluoroethylene. J Thorac Cardiovasc Surg 1998;115:1279-86.

10. Fundarò P, Tartara PM, Villa E, et al. Mitral valve repair: Is there still a place for suture annuloplasty? Asian Cardiovasc Thorac Ann 2007;15,351-8.

11. Kanemoto I, Shibata S, Noguchi H, et al. Successful mitral valvuloplasty for mitral regurgitation in a dog. Jpn J Vet Sci 1990;52:411-4.

12. Boggs LS, Dewan SJ, Ballard SE. Mitral valve reconstruction in a toybreed dog. J Am Vet Med Assoc 1996;209:1872-6.

13. Griffiths LG, Orton EC, Boon JA. Evaluation of techniques and outcomes of mitral valve repair in dogs. J Am Vet Med Assoc 2004;224:1941-5.

14. Kanemoto I, Suzuki H, Taguchi D, et al. Successful surgical repair for severe mitral regurgitation in five small-breed dogs. Vet Surg 2004;33:435-E12 (abstract).

15. Kanemoto I, Taguchi D, Yokoyama S, et al. Open heart surgery with deep hypothermia and cardiopulmonary bypass in small and toy dogs. Vet Surg 2010;39:674-9.

16. Uechi M, Mizukoshi T, Mizuno T, et al. Mitral valve repair under cardiopulmonary bypass in small-breed dogs: 48 cases (2006-2009). J Am Vet Med Assoc 2012;240:1194-201.

17. Nishida M, Kagawa Y, Mizukoshi T, et al. Post-mortem evaluation of expanded polytetrafluoroethylene (ePTFE) used in mitral valve repair in dogs. J Vet Cardiol 2012;14:307-12.

18. Mizuno T, Mizukoshi T, Uechi M. Long-term outcome in dogs undergoing mitral valve repair with suture annuloplasty and chordae tendineae replacement. J Small Anim Prac 2013;54:1047.

19. Kay LH, Egerton WS. The repair of mitral insufficiency associated with ruptured chordae tendineae. Ann Surg 1963;157:351-60.

20. Rittenhouse EA, Davis CC, Wood SJ, et al. Replacement of ruptured chordae tendineae of mitral valve with autologous pericardial chordae. J Thorac Cardiovasc Surg 1978;75:870-6.

21. Kanemoto I, Masumoto T, Ohara K, et al. Canine mitral valvuloplasty by chordal replacement using artificial suture. 20th WSAVA Proc (Japan) 1995:654 (abstract).

22. DeVega NG, Rabago G, Castillon L, et al. A new tricuspid repair. Short-term clinical results in 23 cases. J Cardiovasc Surg 1973:384-6.

23. Becher JR. The surgical treatment of mitral regurgitation. $\mathrm{Br}$ Heart $\mathrm{J}$ 1964;26:513-23.

24. Ishibashi N, Kasegawa H, Koyanagi T, et al. Mechanism of hemolysis after mitral valve repair and new surgical management: prosthetic annuloplasty ring covered with autologous pericardium. J Heart Valve Dis 2005;14:588591.

25. Taguchi D, Kanemoto I, Yokoyama S, et al. Mitral valve replacement with a mechanical valve for severe mitral regurgitation in a small dog. Case Rep Vet Med 2014:1-5. 